\title{
DESAFÍOS DIDÁCTICOS EN LA DISCIPLINA EMPRESARIAL
}

\author{
Henry César Bernal Campos \\ Universidad Pedagógica y Tecnológica de Colombia \\ Colombia
}


Henry César Bernal Campos

JEL: A20, A22, A29

\title{
Desafíos didácticos en la disciplina empresarial
}

\section{Resumen}

Los escasos medios didácticos no responden a la pedagogía activa del conocimiento difundido por los programas de Administración de Empresas. Pese a las exigencias de la política pública dada por las instituciones rectoras de la educación, las acciones se han centrado en el plano verbal y persiste la distancia teoría-práctica. El desafío se da por la dificultad de crear medios didácticos activos para una educación organizacional, que implican los sistemas probabilísticos, autorregulados y complejos de las organizaciones. La falta de comprobación de las teorías ha inducido la observación directa en empresas fuera de la Institución Educativa. El Laboratorio de Empresa como didáctica activa para la educación empresarial, devela algunos caminos para aceptar el desafío de unir teoría-práctica. Las opciones: imaginarse poderoso para dominar la dificultad o aceptar el desafío.

Palabras clave: Laboratorio de Empresa (LdE), Empresa Experimental (EE), Práctica Experimental, Pedagogía Activa, Pedagogía y Didáctica.

\section{Les défis de l'enseignement de la discipline d'entreprise \\ Résumé}

Les quelques supports didactiques ne répondent pas à la pédagogie active de la connaissance diffusée par les programmes d'administration des entreprises. En dépit des exigences des pouvoirs publics en matière de politique publique, les actions se sont concentrées sur le niveau verbal et la distance entre la théorie et la pratique persiste. Le défi est donné par la difficulté de créer des moyens didactiques actifs pour une éducation organisationnelle, impliquant les systèmes probabilistes, auto-régulés et complexes des organisations. L'absence de vérification des théories a entraîné une observation directe dans des entreprises extérieures à l'établissement d'enseignement. Laboratoire de compagnie, en tant que didactique active de la formation en commerce, révèle certains moyens de relever le défi de l'union de la théorie et de la pratique. Les options: s'imaginer d'être puissant pour maîtriser la difficulté ou accepter le défi.

Mots clés: Laboratoire de compagnie, Compagnie expérimentale, Didactique, Pédagogie, Expérimentation.

\section{Challenges in teaching business discipline}

\begin{abstract}
The lack of practical didactic aids does not equal that of the active pedagogy of knowledge diffused by business administration programs. Despite the requirements of public policy given by educational directive Institutions, the actions have been focussed on the verbal plane and hence the theory-practice distance persists. The challenge is given by the difficulty of creating active didactic aids for organizational education, which involves organisation's probabilistic systems, self-regulated and complex. The lack of proof of the theoretical knowledge, has led to direct observation in companies outside the Educational Institution. Hence, the workshop laboratory, as an active aid for business education, reveals some ways to accept the challenge of joining theory and practice. The options, either to imagine yourself powerful to master the difficulty, or accept the challenge.
\end{abstract}

Keywords: Business Lab, Experimental Business, Experimental Practice, Active Pedagogy, Pedagogy and Didactic. 




Artículo de Investigación

\section{Desafíos didácticos en la disciplina empresarial*}

INFORMACIÓN DEL ARTíCULO

Recepción del artículo: 30/10/2018

Concepto de evaluación: $22 / 11 / 2018$

Aceptación del artículo: 12/12/2018
Henry César Bernal Campo**

Universidad Pedagógica y Tecnológica de Colombia, Colombia

\section{INTRODUCCIÓN}

Las instituciones reguladoras de la educación plantean exigencias a todos los sectores pertinentes y se insta al desempeño con calidad de los programas educativos. Dada la importancia de la educación empresarial y la didáctica de carácter activo, se perciben muchos desafíos pedagógicos que precisan confrontar la teoría con la práctica, en la medida que la transición de lo abstracto a lo concreto es tan ardua, como la transición de lo concreto a lo abstracto (Vygotsky, 1995).

En algunas ciencias, la educación ha logrado la demostración de sus teorías y a la par consolida su práctica didáctica. En la psicología de la educación se plantea que los nuevos métodos de la educación y el carácter activo: "...son el espíritu general de las investigaciones pedagógicas, y frecuentemente los mismos métodos de observación, los que al pasar del campo de la ciencia pura al de la experimentación escolar han vivificado la pedagogía" (Piaget, 1987, pág. 167). La historia educativa del tema empresarial, no solo en Colombia, ha pretendido objetivos similares, pero la creación de medios didácticos de carácter activo, son inferiores a los obstáculos reales a superar.

En las reflexiones epistemológicas sobre los temas de la administración, se ha buscado la forma de comprobar las teorías. El peligro de absolutizar el método empírico y verificación de hipótesis, se evidencia en lo expuesto por Salinas (1997) y da fuerza a la evidencia "...al igual que en toda ciencia, en la ciencia de la empresa se deben buscar

\footnotetext{
* Este artículo está relacionado con la investigación de la tesis doctoral "El Laboratorio de Empresa como modelo didáctico de formación multidisciplinar".

**Profesor de la Universidad Pedagógica y Tecnologica de Colombia. Autor de correspondencia: henrycbc@yahoo.com
} 
verificaciones, contrastaciones cruciales que pueden refutar la teoría contrastada" (pág. 49) Pero se presentan dificultades para demostrar las teorías, pues muchas veces es necesario recurrir a la observación empírica, porque:

“...a falta de pruebas de laboratorio, es necesario utilizar las experiencias y observaciones directas de los administradores por lo menos hasta que no se disponga de aquellas, y que los principios de la administración, desarrollados de forma empírica, deberán seguirse utilizando, aunque no se cuente con una comprobación". (Koontz, O’Donnell, \& Weihrich, 1986, pág. 9)

Ante la dificultad para efectuar verificaciones o contrastaciones, llama la atención la última edición no original de Koontz et al., donde los nuevos coautores resaltan la tesis de que "Administrar como práctica es un arte; el conocimiento organizado subyacente a la práctica es una ciencia" (Koontz, Weihrich, \& Mark, 2012, pág. 15) y abandonan la 'prueba' o 'comprobación' de la teoría administrativa.

La 'prueba' o'comprobación' genera un impase difícil de superar. Los desafíos pedagógicos en Administración de Empresas invitan a analizar, generar datos e ideas correlativas, reconocer la importancia de la pedagogía activa, y en especial, los planteamientos de Dewey (1998) en "Cómo pensamos" al observar el proceso educativo. Cuando docentes y estudiantes convoquen la reconstrucción del conocimiento empresarial basado en teoría, el Laboratorio de Empresa $(\mathrm{LdE})^{1}$ ha de permitir contrastar las soluciones planteadas con práctica didáctica activa, a través del currículo y según el interés de la Institución Educativa (IE), se proponen los siguientes niveles: a. educación, básica, media y vocacional, b. educación superior disciplinar y c. educación superior multidisciplinar (Bernal, 2018).

La didáctica tradicional se apoya en esquemas teóricos consolidados en un verbalismo formal, que entre sí, desligan la teoría de la realidad social, económica, ética y acentúan el dilema de la teoría alejada de la práctica didáctica. Otro riesgo del tema empresarial sin medios didácticos adecuados, "...es no utilizarlos por no contar fácilmente con ellos o por no saberlos emplear, con el consiguiente desperdicio de apoyo didácticos para facilitar procesos de aprendizaje" (García \& Malaver, 2000, pág. 102).

El reto planteado para el proceso enseñanza-aprendizaje de la administración, es generar medios didácticos activos, para superar la educación tradicional, que se basa en procesos no observables, excluye al estudiante y retoma teorías sustentadas sólo en palabras con poca comprensión efectiva. Los procesos tradicionales "...no tienen en cuenta la percepción y la elaboración mental del material sensorio que da nacimiento al concepto" (Vygotsky, 1995, pág. 84) y ha de apoyar el proceso enseñanza-aprendizaje. Superar el obstáculo, permite actuar en el proceso básico de la formación del concepto científico, integrado a la acción.

Los medios didácticos que se logren normalizar para cada Laboratorio de Empresa, podrán ser acreditados según el nivel de observación alcanzado. Con este objetivo la Escuela de Administración de Empresas de la Universidad Pedagógica y Tecnológica de Colombia

1. Laboratorio de Empresa (LdE): el Laboratorio de Empresa (LdE) es una didáctica para trabajar el conocimiento, habilidades y destrezas del tema Empresarial como alternativa viable de prácticas activas, experimentales a través del currículo para Administración de Empresas. 
(Uptc), ha orientado una Empresa Experimental (EE)², que permite contrastar varias soluciones planteadas en los temas estudiados a través del currículo. Para el desarrollo de una Empresa Experimental se recomiendan las siguientes fases: I. Procesos manuales, II. Informatizar procesos administrativos y operativos, III. Empresa Experimental Inteligente y IV. Comunidad Virtual inteligente (Bernal, 2018).

\section{REVISIÓN DE LITERATURA}

Se observa la normatividad emitida por los entes rectores de la política educativa nacional, las particularidades del devenir educativo en los programas de Administración de Empresas y sus desafíos.

\section{Políticas en educación superior}

Para el acompañamiento pedagógico a través de los años, en la enseñanza de las ciencias naturales se crean sus respectivos recursos didácticos y responden al desarrollo del saber. A la par del avance teórico, se da la didáctica respectiva y genera unas exigencias públicas y un autocontrol, en quienes desean difundir o apropiarse del conocimiento de dichas teorías. Su desafío no se da sólo en lo teórico, ni sólo al inicio académico, se logra para los diferentes niveles del sistema educativo como lo exigen diferentes órganos de política educativa a nivel nacional.

Los programas en Administración de Empresas y afines, han pretendido responder con métodos pedagógicos y medios apropiados, que de igual manera, equilibren la teoría y práctica respectiva, pero la ausencia de éste equilibrio, se presenta como una falsa dicotomía intrínseca de la disciplina empresarial (Laufer, 2006). En su aspiración por proponer una formación pertinente, las instituciones de educación de diferente nivel, han adoptado medios didácticos como: simulaciones, proyectos, juegos, casos, visitas, o las tradicionales prácticas de final de carrera. Sin embargo, no se ha podido iniciar con el acompañamiento didáctico articulador de cada tema, iniciando por demostrar lo sencillo, sino que se ha recurrido a una práctica final en las empresas. Como alternativa, históricamente se aludió a la microempresa, después fue la incubación empresarial y últimamente, el arquetipo empuñado es el emprendimiento (genérico) que no obliga a usar el conocimiento organizacional.

\section{Ministerio de Educación Nacional (MEN)}

Con el objeto de cumplir los fines de la educación en Colombia, la Ley 30, en el Artículo 6, literal c, legitima "Prestar a la comunidad un servicio con calidad, el cual hace referencia (...) a los medios y procesos empleados..." (1992, pág. 1). Por ello, se reglamentó la anterior

\footnotetext{
2. Empresa Experimental (EE): la Empresa Experimental de la Uptc, es una organización real, legalmente constituida como Cooperativa de los Estudiantes de Administración de Empresas de la Uptc, denominada Coolíderes. Germina como un organismo vital de la didáctica, para diferentes temas sustentados con Laboratorio de Empresa. Su objeto social es colaborar con la práctica transversal del programa de Administración de Empresas, al poner su estructura al servicio de la academia, para ser observada desde el Laboratorio de Empresa. Su actividad económica es comercial por ser de menor complejidad. El monto del aporte, el tamaño de la EE y el riesgo económico mínimos, inducen una toma de decisiones sencilla y permite al estudiante centrarse en su formación.
} 
Ley con el Decreto 1403 de 1993 y en el Artículo 1, numeral 12, se planteó establecer los "Recursos específicos para desarrollar el programa: -Número de aulas previstas. -Laboratorios y Equipos. -Lugares de práctica..." (MEN, 1993), requeridos para relacionar la teoría, con pedagogía y práctica didáctica.

Dada la preocupación por la calidad de la educación en Colombia, el Ministerio de Educación Nacional (MEN) estableció condiciones mínimas para lograrla, con los Decretos 0808 (MEN, 2002a) y 2566 (MEN, 2003a). El Instituto Colombiano para la Evaluación de la Educación (ICFES), advirtió del riesgo de cursar estudios de educación superior en instituciones de dudosa calidad y además, tener en cuenta "las ayudas didácticas que ofrecen, las instalaciones locativas..." (ICFES, 2002, págs. 2-9) de las instituciones.

El Decreto 938 (MEN, 2002b) establece estándares de calidad en programas profesionales de pregrado en Administración, y la Resolución Número 2767, Art. 1, especificó que "Las denominaciones académicas de los programas de Administración serán de tres tipos: básicas, integración de dos o más básicas, y otras denominaciones..." (MEN, 2003b, pág. 1). Se insiste "...no se va a permitir que instituciones universitarias que no cumplan con los requisitos vayan a funcionar" (MEN, 2006, pág. 3B).

La calidad de la educación que reciben los estudiantes, es un objetivo del MEN. Uno de los instrumentos de seguimiento es la presentación del examen SABER-PRO, como herramienta de observación de los planes de mejoramiento de la calidad y del proceso de enseñanza.

Para compilar las normas preexistentes que reglamentan el Sector de la Educación, el MEN (2015), expidió el Decreto 1075 y sigue exigiendo precisar los medios para la práctica pedagógica. Precisamente, normó una serie de condiciones para obtener el Registro Calificado y vela por la organización de actividades académicas como: laboratorios, talleres, etc. En la Investigación, se preocupa por la ambientación de una actitud crítica, la promoción de la formación investigativa y sus objetivos. Se espera el impacto de los programas en la sociedad y el trabajo con la comunidad. Entre otros, confirma la necesidad de disponer de medios educativos como "...equipos y aplicativos informáticos sistemas de interconectividad, laboratorios físicos, escenarios de simulación virtual de experimentación y práctica, talleres con instrumentos y herramientas técnicas e insumos" (MEN, 2015, pág. 334). Adicionalmente, pide garantizar infraestructura física, entre otras "...laboratorios y espacios para la enseñanza, del aprendizaje (...) la metodología y las estrategias pedagógicas, las actividades docentes, investigativas” (pág. 334). Las anteriores condiciones del Registro Calificado requeridas por el MEN (2015), exigen disponer de medios para desarrollar la pedagogía.

\section{Consejo Nacional de Acreditación (CNA)}

En la medición de los lineamientos para la Acreditación de Programas de Pregrado, desde 1996 (CNA) ya se propone observar los medios de apoyo docente. La "Característica n” 26. Recursos de apoyo docente" (CNA, 2013), propone seis aspectos a evaluar, entre los que genéricamente para todos los pregrados y pregunta por medios de apoyo muy específicos para la implementación del currículo, tales como: a) talleres, b) laboratorios, c) equipos, d) materiales, e) estaciones y demás. La falencia de medios de apoyo didáctico activo para los docentes de los programas de Administración de Empresas, nuevamente se hace visible. Los anteriores lineamientos, igualmente permiten evaluar la existencia de medios con que se desarrolla la pedagogía en cada programa de Administración de Empresas, según el enfoque (Díaz, 1998), los modelos y los medios didácticos (Medina \& Salvador, 2009). 


\section{En las universidades}

Si en las disciplinas propias de la educación, la pedagogía y didáctica parecen palabras más que conceptos (Zambrano, 2006), y en unos planes de capacitación pedagógica para profesores de ciencias en universidad, "...lo pedagógico y lo didáctico han carecido de importancia” (Gallego \& Pérez, 1997, pág. 269). En los programas en Administración de Empresas, los conceptos pedagogía y didáctica adolecen de interés para la enseñanza de éste conocimiento, coexiste, además, un escaso desafío en la capacitación y actualización pedagógica. No obstante, el clamor por una solución adecuada a los conflictos sociales, centra la esperanza en la empresa y la universidad. Paralelamente, en Administración de Empresas se evidencia el reto y se argumenta "...no se justifica un conocimiento que no sabe transmitirse y tampoco se justifica transmitir bien un conocimiento sin validez" (García \& Malaver, 2000, pág. 89); este argumento exige una pedagogía con su didáctica pertinente.

\section{Realidad específica de la disciplina empresarial}

En Colombia en 1985 existían 36 programas de Administración de Empresas formalmente tramitados ante el ICFES (Bernal \& Berdugo, 1985). En 1996 se reportaron 277 diferentes programas de pregrado (ICFES), cuyo nombre usaba la palabra administración en alguna parte de su denominación; de los anteriores programas, 154 iniciaban con la palabra administración. De los 277 programas, 87 se llamaban Administración de Empresas y estaban debidamente aprobadas por el ICFES (Bernal, 1997).

Para el año 2002, en el ICFES se registraron 256 programas de pregrado cuyo nombre usaba la denominación específica 'Administración de Empresas'. El dato anterior no incluye programas técnicos, tecnológicos, especializaciones, maestrías y doctorados que en su denominación contienen la palabra administración. Según el Consejo Profesional de Administración de Empresas (CPAE, 2013, pág. 60), se observa la oferta de 687 programas autorizados en nivel de formación universitaria. Sin incluir programas afines, ni otros niveles de educación, en 2015 se graduaron 18.101 Administradores de Empresas (MEN, 2016), es decir 10.15\% del 100\% de graduados del país. No obstante, lo expuesto anteriormente no es el problema.

Ha aumentado el número de programas en cuestión pero no sus prácticas a través del currículo, siendo un fenómeno global (Ramírez, 1994). En Colombia y seguramente en otros países, "Se ha considerado que la proliferación de la enseñanza administrativa no obedece a la importancia que realmente tiene, sino, más bien, al bajo costo y facilidad en su programación de estudio" (Palomar, 1986, pág. 40). Así, la organización educativa "ha soportado la proliferación de programas de administración de pregrado y posgrado..." (Malaver \& López, 2016). Dicho desequilibrio, contribuye a generar el llamado "No a las universidades de 'garaje"' (MEN, 2006), al anunciar que impedirá el funcionamiento de las instituciones universitarias que incumplan los requisitos. Se espera que el ciberespacio no facilite la proliferación de 'universidades de garaje virtual'.

En la medición de grupos Colciencias (2013), se clasificó la Administración en las Ciencias Sociales Aplicadas. En el posterior documento de medición de grupos (Colciencias, 2015a), a la especialidad del Área de conocimiento para la "...carrera de mayor demanda" (MEN, 2012, págs. 10-11) se le llama Negocios y Management, probablemente "...para que esté más a tono" (Max-Neef, 2013, pág. 5). Curiosamente, el área de actuación 'Administración' se elimina, excepto para "Administración de Hospitales" y "Administración Pública" (Colciencias, 2015b, págs. 196-197). 
En administración la intención didáctica es desconocida y no todos los estudiantes acceden a la Práctica. Entre los dilemas del currículo, persiste el desbalance entre la teoría y la práctica para que la gestión pedagógica del docente, garantice unas experiencias prácticas del estudiante (CPAE, 2017). Llama la atención la afirmación "Si bien la práctica surge como una exigencia del sector productivo, la investigación surge como una exigencia normativa" (CPAE, 2017, pág. 37), al dejar en entredicho la integralidad y pertinencia del factor de procesos académicos, a evaluar por el CNA. En la perspectiva de los empresarios, un punto suficientemente crítico "...es la gran brecha que se presenta entre la percepción de los empleadores y los propios estudiantes (págs. 45-46).

\section{El devenir educativo y desafíos en Administración de Empresas}

Una adecuada y pertinente educación en los programas de Administración de Empresas, ha de acercar la teoría y su práctica respectiva, hasta ahora distantes. El abismo se presenta en todos los niveles del sistema educativo afín al conocimiento empresarial, dada la dificultad de generar medios didácticos articulados a una realidad, que superen la pedagogía tradicional, en la que prevalece la memorización de teorías sin contexto.

\section{La pedagogía en Administración de Empresas}

En las reflexiones expuestas para los programas de Administración de Empresas, difícilmente se considera que ellos deberán disponer de medios didácticos de enfoque positivista, interpretativo y de ciencia aplicada (Díaz, 1998), para que el docente o discente pueda seleccionar lo pertinente. El problema se genera por el aislamiento entre teoría y práctica de lo que se considera conocimiento organizacional. Este conflicto, encadena muchos desencantos en empresarios, profesionales y estudiantes, lo cual a la vez, despierta la atención de los investigadores. Los interesados en la educación organizacional hacen intervenciones pedagógicas y didácticas, con acercamiento parcial de teoría y práctica pero con resultados insatisfactorios e inadecuados a las perspectivas exigidas para Administración de Empresas. El interés por una formación organizacional con legitimidad social, sigue alejado de resultados prácticos. Al referir un proceso dado en la Universidad Javeriana desde el Comité para la Formación Integral del Docente de administración, se expresa lo que se puede hacer extensivo en la enseñanza universitaria para todos los programas de administración, al exponer que:

Los problemas de la enseñanza, la pedagogía y la didáctica ocupan un lugar marginal en el ámbito académico (universitario), cuando no le son 'totalmente ajenos' (...)

En la evaluación del aprendizaje de los estudiantes predomina la memorización de datos (...)

Por lo general, los profesores son intelectualmente competentes, pero existen fallas en lo pedagógico-educativo... (García \& Malaver, 2000, pág. 89)

En la enseñanza de los temas empresariales surge la inquietud que genera "...problemas terminológicos, principalmente por las variedades de significados o polisemia que puede tener un mismo término, la proliferación injustificada de sinónimos y de términos y su falta de precisión, todo lo cual, según muchos autores, sólo propicia confusión" (Ríos, 
2008, pág. 51). Se genera una educación que difunde cantidad de teorías empresariales, sin exigir medios didácticos activos para ayudar a depurar los conceptos. En un proceso parcial, se memorizan palabras aisladas, e inicia su uso vago "...como medios de comunicación mucho antes de alcanzar el nivel de los conceptos característicos de pensamiento totalmente desarrollado" (Vygotsky, 1995, pág. 87).

La práctica transversal que pedagógicamente se usa en otras ciencias, en administración adolece de criterios, esconde su posibilidad, mimetiza su superficialidad y no expone la dificultad para crear ese apoyo docente. Se desconoce su importancia en situaciones de enseñanza; no está disponible para los diferentes niveles educativos y con frecuencia se usa la palabra "práctica" como un término genérico de connotación única. Igual que otras ciencias, "...en algunos casos se recurre a una aplicación mecánica de la teoría a la práctica y en otros se abandona lo teórico, asumiendo a la práctica como un único criterio de verdad" (Barnechea, González, \& Morgan, 1994, pág. 83). La práctica “...implicaría detectar y confirmar desajustes entre teoría y realidad, y convertirlos ¿por qué no? en fuente de generación de nuevos conceptos o teorías" (Malaver \& López, 2016, pág. 156).

El acercamiento a la palabra "práctica" evidencia ligereza y ambigüedad en su uso: la práctica solamente verbalizada, TIC simulada como práctica, prácticas abusivas, emprendimiento como práctica, práctica pseudo-profesional, la práctica académica de fin de carrera y la práctica profesional. Así se incrementa la polisemia y los errores en el uso adecuado de los términos. La carencia de prácticas en el plan de estudios y práctica en empresa, sin didáctica previa, se solapan entre los otros usos como si fuesen el problema y la solución se sigue dilatando (Bernal, 2016).

Las instituciones educativas adolecen de medios de apoyo didáctico activo, como primer acercamiento práctico en la construcción del conocimiento de un Administrador de Empresas. Dicha falencia debe ser el problema inicial a enfrentar en la profesión y la disciplina al tratar de superar los errores heredados de la formación, visibles en la práctica de fin de carrera, o lo vivido en la empresa al desempeñar la profesión. En esta última, se acusa la falta de práctica durante la formación, y la educación organizacional, acepta el dilema teoría sin práctica y faltan criterios para proponer categorías didácticas, su fundamentación, su estructura y los fines de cada una de ellas (López \& Sanabria, 2011).

\section{¿Qué se ha hecho para mejorar la educación empresarial?}

En el esfuerzo por encontrar soluciones para acercar teoría y práctica, se presentan muchas opciones. En un diagnóstico se planteó que para “...desarrollar la práctica hacia la mitad o (...) en las (...) tres cuartas partes de la carrera, es complicado porque le falta al estudiante una serie de conocimientos" (Ramírez, 1994, pág. 4), implica que, ninguna profesión lograría hacer prácticas desde el primero hasta el último semestre de formación, porque de igual manera a los estudiante de otras profesiones, también les faltarían toda una serie de conocimientos. Si “...en Italia, España y Suiza, la práctica tiende a hacerse ahora más en el postgrado que en el pregrado" (Ramírez, 1994, pág. 7), se confirma que el problema no sólo se presenta en Colombia. En otros países, tampoco ha disminuido la brecha teoría práctica del aprendizaje organizacional y afines, si no, en Colombia dicho modelo ya se habría implementado.

Unas disciplinas (como medicina, ingeniería y ciencias naturales) pueden lograr prácticas desde el primer semestre y son de carácter obligatorio. Además, son experimentales, 
con sus propios objetos sensorios como medios didácticos activos, transferibles a cualquier programa y han logrado desarrollar la práctica a través del currículo. Sin embargo, los programas como Administración de Empresas y afines, no han podido implementar las prácticas transversales al plan de estudios, con didáctica activa para cada semestre o asignatura.

A la carencia de práctica se suma la ambigüedad de la supuesta teoría. Es ilógico que a todo escrito en administración se le llama teoría como si cualquier "...impreso en los libros, por ese simple hecho es teoría, creencia muy divulgada entre alumnos y docentes de la administración" (Ríos, 2008, pág. 41). A diferencia de otros programas, en administración se difunden opiniones de empresarios influyentes o comentarios de noticieros. La teoría no probada, acrítica, con "...la Universidad se ha transformado, quizás ingenuamente, en cómplice” (Max-Neef, 2013, pág. 1) del incremento de reflexiones, de "...corte disciplinario-convencional, no justificado, ha terminado por transformarse en contenidos teóricos con un alto grado de cohesión interna" (Zemelman, 2009, pág. 54). Así, notables docentes, estudiantes e investigadores acaban por abandonar la "prueba" o "comprobación" de las teorías administrativas. Sin práctica, logran acomodar la realidad social a ese abuso verbal, en detrimento de una mejor educación empresarial con pertinencia organizacional y social. Un ejemplo, para la toma de decisiones basada en datos, señala "...un desempeño muy bajo en el manejo del área financiera" (Romero, Castelblanco, \& Bastidas, 2013, pág. 34). Si esta área regida por las normas internacionales de información financiera poco usa el conocimiento, en otras áreas con mayor libertad de omisión el uso del conocimiento debe ser menor.

\section{Salidas didácticas de la disciplina empresarial}

Algunos profesores, estudiantes e investigadores "...guardan en común la preocupación por esclarecer la problemática presente en la formación de Administradores" (Agudelo, 2007, pág. 12). Si en las disciplinas propias de la educación, "...los conceptos pedagogía y didáctica han divagado entre el olvido y el reconocimiento” (Zambrano, 2006, pág. 99) en otros campos de la educación como el aprendizaje organizacional poco se ha investigado. Encontrar medios idóneos para disminuir el distanciamiento entre la una y la otra, ha sido tarea confusa y paralizante. Académicamente la pedagogía y la didáctica adolecen de la atención necesaria, o se olvidan porque no se reconoce su importancia, además, ha aumentado el conformismo y ante cualquier teoría todo se vale como práctica.

Es patente el dilema de teoría y práctica en la formación en administración pues no se ha logrado ésta unión. La distancia, es consecuencia de la dificultad para crear medios didácticos activos. La famosa frase 'No hay nada más práctico que una buena teoría' es pertinente, pero el aprendizaje de la administración, como mínimo, debe invitar a probar sus postulados y procedimientos básicos y, con ello, preparar la transferencia de conocimiento a la acción empresarial. No llevar a la práctica los conocimientos elementales implicaría aceptar que lo hecho empíricamente en las organizaciones es mejor que las reflexiones teóricas.

El desafío a nivel didáctico de una asignatura o tema, para el desarrollo del concepto verdadero ha sido indeterminado en la educación empresarial y tiende a dejar el pensamiento en pre-conceptos difusos (Vygotsky, 1995). De lo difuso se encuentran varias muestras en las ciencias afines, derivadas de la ausencia de didáctica especializada, que también revelan la gravedad del problema. La situación se torna más grave, porque, en Administración de Empresas Koontz et al. argumentan: 
...que la administración es la más inexacta de las ciencias sociales. Es cierto que las ciencias sociales, y en particular la administración, se ocupan de complejos fenómenos sobre los cuales existe poco conocimiento, y es cierto también que la estructura y conocimiento del átomo son mucho menos complejos que la estructura y el comportamiento de grupos de personas, incluyendo los internos y externos a la empresa. (1986, pág. 9)

Otro ejemplo, con mucha resignación, para uno de los medios pedagógicos tradicionales se afirma:

El método de casos es una técnica de simulación. Puesto que el alumno no puede manejar una empresa de manera experimental (como un estudiante de medicina aprende a hacer cirugía en cadáveres o como un estudiante de ingeniería maneja reacciones químicas o físicas en el laboratorio), el método de casos busca simular una realidad donde el alumno pueda poner sus conocimientos 'en práctica'" (Chiavenato, 2007, pág. 18)

Sin embargo, el método de casos está lejos de ser la solución (Aktouf, 2000). Por esta razón para Salinas "...la teoría de la empresa, debe construirse a partir de la realidad, y se conviertan en instrumento de conocimiento que elimine el falso dilema entre teoría y práctica" (1997, pág. 80). Ahora, se trata de encontrar medios que apoyen la reconstrucción didáctica de la teoría desde la compleja realidad, que elimine el aparente antagonismo entre teoría y práctica de la Administración. Los estudiosos de la ciencia organizacional, no se deben conformar o resignar con los métodos pedagógicos pasivos que dejan insatisfechos a un sinnúmero de estudiantes, profesores, profesionales, empresarios y a una sociedad inconforme y ansiosa de buenas soluciones.

Pero antes que juzgar cualquier otra opción, es que los dilemas siendo la consecuencia se ven como el problema a resolver, distraen la atención y ocultan la causa, ¿Cómo unir teoría probada con práctica didáctica, que responda adecuadamente a la educación empresarial? Si se pretende superar el incomprensible camino de una didáctica transversal, los medios didácticos deben asimilar la realidad, dominar el conocimiento y hacer menos difusa la teoría. El desafío es lograr una práctica académica con el rigor de la teoría administrativa, en vez de que se repelan como el agua y el aceite, mal usados (Malaver \& López, 2016).

La explicación de empresa desde la Teoría de Sistemas, la considera como un sistema complejo y probabilístico, con variables (controlables y no controlables), con capacidad de modificarse y que requiere autorregulación (Chiavenato, 2007). Desde este punto de vista se plantea que la teoría de sistemas llegó a la teoría general de la administración, para afianzarse en la informática y hacer surgir un nuevo mundo organizacional, donde la técnica y tecnología proveen soluciones al crecimiento empresarial, como sistemas dinámicos y compleja realidad. No obstante, un sistema empresarial que pretenda definir íntegramente una realidad organizacional, siempre generará desafíos por falencias en información, las muestras no siempre serán representativas y continuamente se obstruirá el conocimiento absoluto de todas las variables (Chiavenato, 2007). Por ello "...Los académicos estamos llamados a la investigación disciplinar (...) debe convocarnos desde la perspectiva de la responsabilidad, es decir, desde una posición ética y no solamente productiva" (López F. , 2005). Además, en la educación empresarial se debe exigir una práctica didáctica, que en medio de la sencillez académica, genere infinitas interacciones y los datos sistémicos requeridos, cuenten con una totalidad económica, social y ética según el tema a cursar y probar. 


\section{- Los desafíos en Administración de Empresas}

A la advertencia ¿cuáles son "las ayudas didácticas..." especializadas y desarrolladas que ofrecen los programas de temas empresariales en Colombia? Si se evaluaran los medios especializados para cada profesión, como lo expresó Palomar desde 1986 (pág. 40) ¿Qué se pediría a carreras que sólo cuentan con un bajo costo y son fáciles de programar?

Según Montoya: el 39 \% “...de los empresarios manifiesta tener problemas para encontrar (...) personal contable y de finanzas, obreros y operarios de producción” (2010, pág. 4). Seguramente, las pruebas SABER PRO podrán medir los conocimientos memorísticos de los universitarios en el área del conocimiento empresarial, pero no pueden evaluar su saber práctico.

La exigencia del MEN está ligada a la calidad de la educación y al riesgo de proliferación inadecuada, pero, la falencia de la didáctica empresarial persiste en cualquier universidad, no en una u otra. Sucede lo mismo en las Instituciones de Educación básica y media con especialidad afín a la formación empresarial. De ahí la importancia de acciones conjuntas como la Estrategia nacional de educación económica y financiera del Ministerio de Hacienda y Crédito Público (MHCP, 2010)

La respuesta de la pedagogía organizacional tradicional se justifica en lo teórico, sin articular su práctica; unos semestres se acompañan con visitas, casos, juegos empresariales, o con simulación ${ }^{3}$. Al final de la carrera, en la mejor de las situaciones se desarrolla el semestre de Práctica Empresarial. Se desconocen los enfoques del pensamiento didáctico (Díaz, 1998) y los modelos didácticos (Medina \& Salvador, 2009) adecuados para el docente. La pedagogía con didáctica activa para respaldar cada teoría, difícilmente se concibe; de darse estará desarticulada.

Dosificar teoría y práctica didáctica es una necesidad curricular y conflicto, porque el tiempo en el aula parece insuficiente para la descomunal teoría administrativa, que denotan la persistencia dominante del modelo pedagógico tradicional, centrado en la verbalización a memorizar, e incapaz de propiciar el uso práctico de lo aprendido. La pedagogía activa ha de permitir: dosificar la acción (Londoño, 2008), apoyar la labor directa del docente, acompañar al estudiante en actividades independientes de estudio, prácticas, u otras (MEN, 2015), que desarrollen medios didácticos transversales al plan de estudios y avanzar con una construcción lógica del conocimiento.

\section{MÉTODO}

El método aplicado recurre a un enfoque mixto, con metodología investigación acción y sistematización de experiencias. Unos procesos técnicos obedecen la norma, son cuantificables y a la vez, se articula la interpretación de variables cuantitativa y cualitativa.

\section{Investigación acción}

La investigación acción “...significa planificar, actuar, observar y reflexionar más cuidadosamente, más sistemáticamente y más rigurosamente de lo que suele hacerse en

3. Simulación: popularmente usada como imaginar datos o fingir acción. 
la vida cotidiana" (Kemmis y McTaggart, 1992, p. 16), que al contar con el Laboratorio de Empresa y la Empresa Experimental, la acción de los estudiantes practicantes se da en un ambiente real.

La actividad económica de la Empresa Experimental debe ser sencilla, pues se busca que los estudiantes participantes centren su atención en las ciencias auxiliares de la administración, en el saber de ella misma y que el conocimiento de los procesos de la actividad económica solo sea tangencial.

\section{Sistematización de Experiencias}

La sistematización de experiencias como metodología para la "...producción de conocimiento crítico desde la práctica, ha ido adquiriendo más y más relevancia en las experiencias de educación (...) Muchas veces confundida con la mera recopilación de datos o con la narración de eventos" (Jara, 2012, pág. 56). En este sentido, una función del Laboratorio de Empresa es sistematizar las vivencias de los laboratorios experimentales y de la observación facilitada por la Empresa Experimental, para reconstruir de ella y de los experimentos, los procesos cotidianos comparables a la teoría, que valide el conocimiento cursado, practicado, o detectar las variantes en contexto.

La sistematización de experiencias promueve el aprendizaje desde la acción reflexionada, revelar variaciones significativas y confrontar la teoría cursada, con respecto al uso u omisiones observadas en la Empresa Experimental, desaprender lo que se entendía o ratificar lo aprehendido. Es importante reflexionar las experiencias adquiridas durante los experimentos y preexperimentos y de ellos, razonar que a las prácticas académicas “... le subyace una teoría que debe ser explicada de acuerdo al contexto en el cual se produce la experiencia" (Mejía , 2013, pág. 20).

\section{RESULTADOS}

Desde la acción participación y la sistematización de experiencias del Laboratorio de Empresa y la Empresa Experimental, se manifiesta una propuesta para acercar la teoría a la práctica de los programas de Administración de Empresas.

El desafío para disponer de medios didácticos activos, que pedagógicamente acompañen el acercamiento de la teoría a la práctica, empieza a superar la dificultad que implican crear y simultáneamente articular: sistemas probabilísticos, autorregulados y complejos, pertinentes a la educación organizacional.

El laboratorio de Empresa y la Empresa Experimental, han permitidoconsolidar espacios académicos de comprobación experimental, preexperimental, cuasiexperimental, o la exploración (Hernández, Fernández, \& Baptista, 2010).

La fundamentación de la estructura didáctica taxativa, interpretativa y ciencia aplicada, se sigue consolidando y la sistematización de experiencias gira en torno a la pedagogía activa, que se apoyan en el Laboratorio de Empresa y en la Empresa Experimental. 


\section{ANÁLISIS Y DISCUSIÓN}

La falta de comprobación de las teorías cursadas en los programas de Administración de Empresas, ha inducido la observación directa en empresas fuera de la IE, con los inconvenientes pedagógicos y didácticos que limitan el pensamiento racional y crítico. No obstante, al articular el enfoque didáctico taxativo, interpretativo y ciencia aplicada con el Laboratorio de Empresa y la Empresa Experimental, se han podido categorizar múltiples medios didácticos, que ordenan el pensamiento. Los enfoques didácticos ordenan datos e ideas correlativas a la siguiente propuesta inicial de categorías: Actividades en Equipo, Actividades en Grupos, Documentos, Entorno, Instrumentos Físicos, Instrumentos Abstractos, Materiales Abstractos, Mediadores Externos y Mediadores Internos entre otras.

\section{Cómo Pensamos}

Al reflexionar sobre los medios didácticos para la educación empresarial con pedagogía activa, se puede usar la obra de Dewey: Cómo pensamos (1998). Pero, es iluso iniciar la formación de un pensamiento reflexivo con una base práctica, que depende de una realidad empresarial foránea a la IE y al estudiante. Ha de hacerse por medio de una Empresa Experimental, en la que es posible empezar con una práctica didáctica, dosificada desde un Laboratorio de Empresa; implica que sea una actividad simple, pero, de hecho, no hay nada simple (Morín, 2004). El desafío está en conseguir datos e ideas correlativas, como medios didácticos activos para apoyar una formación disciplinar, interdisciplinar y multidisciplinar idónea. De otra forma “¿Cómo pensar la didáctica empresarial con soluciones magistrales que excluyen más datos e ideas, que las que integran?” (Bernal, 2016, pág. 25).

Con la Empresa Experimental se logra disponer de una totalidad de objetos agrupables en muchos campos de objetos para observar una compleja realidad mutable. Las variables son relativamente controlables según las capacidades del investigador, los temas a observar y la presencia de teorías transferidas a la Empresa Experimental. Así es posible abordar variables empresariales e iniciar el pensamiento en complejos (Vygotsky, 1995), que ameritan la investigación formativa y luego la científica. Por ello, el material recomendado para experimentar, puede empezar por probar la teoría normativa social básica (Max-Neef, 2013), avanzar según el nivel de formación y la capacidad experimental.

Generar y observar un ambiente organizacional experimental como apoyo didáctico activo, implica dificultad y asombro. Al aceptar el desafío, Dewey (1998) propone unas fases pedagógicas para desentrañar un ambiente difuso. Acercar teoría y práctica, radica en observar y reflexionar los medios con que se cuenta, y los fines que se pretende alcanzar. Muchos desisten, o se conforman con alternativas estériles. La información y reflexión de los medios didácticos aportados demanda esfuerzo y la mente tiende a rehuir de tales molestias. La pedagogía activa con Laboratorio de Empresa, invita a desafiar situaciones reales y a usar el ciclo repetitivo de Lewin (1946) de planear, actuar, observar y reflexionar (Kemmis \& McTaggart, 1992).

\section{El pensamiento reflexivo}

Para el desarrollo del pensamiento reflexivo es indispensable tener en cuenta ciertos requerimientos (observar y sugerir) y factores correlativos (información y reflexión). 


\section{El pensamiento reflexivo requiere observar y sugerir}

Al pretender la observación de datos e ideas en una empresa inventada, fluyen a la mente propuestas especulativas, que luchan entre ellas; igual, al intentar proyectos en empresas foráneas a la IE. En contraste y desde la clase, enfrentar proyectos reflexivos en la Empresa Experimental, también vienen a la mente alternativas que compiten entre ellas, pero con datos e ideas reales. Así, las sugerencias que compiten entre sí, exigen decisiones, acompañadas de implicaciones en esa aparente sencillez de la Empresa Experimental. Las observaciones llegan seguidas de medios favorables, obstrucciones reales y desafíos teóricos. El modo decidido de acción práctica, debe estar respaldado con sugerencias viables; lo contrario, impide el pensamiento reflexivo y se provoca la paralización del juicio.

Las variables favorables y obstrucciones son la constante del mundo empresarial, que ameritan observar y seleccionar medios, acordes con un sistema de valoración. Con empresas externas, es difícil que un estudiante logre llegar a las variables, o intervenir en ellas. Una Empresa Experimental facilita y se puede observar, seleccionar, proponer, e incluso actuar; ejemplo: los ingresos, la utilidad, los costos, los gastos, la pertinencia, la accesibilidad, etc. Hasta es factible que alguna idea se implemente en la Empresa Experimental, por ser real y en la cual, actúan los estudiantes. Verbigracia, ampliar el personal: la idea genera reflexión y los datos siguen latentes, con necesidad de comprobación o corrección, el pensamiento reflexivo observa y sugiere nuevas alternativas.

\section{El pensamiento reflexivo requiere factores correlativos: información y reflexión}

Para Dewey "El termino técnico que designa los hechos observados es datos" (1998, pág. 100) y en una realidad empresarial se deben reflexionar. Las sugerencias relacionadas con los datos, proponen soluciones posibles que son las ideas o alternativas por las que hay que tomar decisiones empresariales. Así, "Datos (hechos) e ideas (sugerencias, soluciones posibles) constituyen los dos factores indispensables y correlativos de toda actividad reflexiva" (Dewey, 1998, pág. 101), para el análisis de variables y las inferencias que surgen de las propuestas ideadas. Los datos observados e ideas sugeridas constituyen los medios de apoyo didáctico activo, que hay que relacionar, reflexionar, e interpretar con la teoría a comprobar.

El rigor de las observaciones e inferencias podrán ser experimentadas e implementadas en el Laboratorio de Empresa como práctica didáctica; al hacerlo o no hacerlo, distintos estudiantes aprenden de las acciones, omisiones, reflexiones y observaciones de los otros aprendices (Piaget, 1987). Por esta razón se requiere una investigación formativa, "...que permita tender puentes entre académicos y practitioners" (Malaver, 2016, pág. 66) y reflexionar las sugerencias teóricas, con los datos reales que ofrece la Empresa Experimental como "Puentes entre la teoría y la práctica" (Pinzón, 2016, pág. 5).

La acción de los experimentos trasciende en la realidad concreta y se aproxima a la mínima práctica durante la formación. La idea empresarial, ahora inferida sobre datos sólidos, requiere doble comprobación: el proceso de formación de la idea y el proceso de su comprobación con la puesta en práctica. La prueba se hace real, con datos de la Empresa Experimental y confirma o niega la teoría. Si la comprobación se hace sobre lo imaginario, sin correlación, la inferencia será especulativa, y por vana, seguramente no se seguirá analizando. 
La experiencia se enfrenta a una teoría que exige reflexión. Los ejemplos organizacionales básicos, se defienden con datos e ideas de magnitud relativamente controlable, e información relacionable. Los datos se deben usar correlacionados y las ideas solitarias, no son más que especulaciones; recurrir a datos e ideas por separado, descontextualiza y sigue generando tensión e insatisfacción. Datos completos de la Empresa Experimental para reflexionar con ideas correlativas, generan nuevas reflexiones, que siguen dando respuesta a posibles prácticas pasadas, presentes o futuras.

\section{Actividad reflexiva en el Laboratorio de Empresa y la Empresa Experimental}

La función de la actividad reflexiva para el acercamiento de la teoría y la práctica del tema empresarial, reclama factores correlativos e incita enfrentar las observaciones con la profundidad necesaria. Desde el pensamiento reflexivo de Dewey (1998) como camino para enfrentar los temas que entrañan dificultad, se distinguen dos límites de la actividad del pensamiento: un extremo desconcertante, confuso y enmarañado; el otro extremo, finalizado con una situación clara, sin dudas. Dewey a la primera la llama pre-reflexiva y al final lo llama post-reflexiva con exaltación del dominio, satisfacción y goce.

Lograr la reconstrucción del pensamiento organizacional con factores correlativos, observación completa, vivificar el juicio, y estimular la sugerencia de soluciones alcanzables, además requiere reflexionar su teoría "...en torno a la función analítica de la totalidad" (Zemelman, 2009). Lo expuesto se logra al viabilizar experiencias en el Laboratorio de Empresa con la totalidad de la Empresa Experimental. La pre-reflexión, más que articular una práctica, exige reflexionar y entender su teoría en una totalidad en movimiento. Para llegar al límite final post-reflexivo del desafío didáctico del aprendizaje organizacional, se desglosa el proceso en cinco etapas, no tan secuenciales ni fijas, como lo precisa Dewey (1998):

\section{Primera Etapa: Las sugerencias}

Por naturaleza el ser humano hace sugerencias; se refieren solo a ideas poco elaboradas que permiten continuar las acciones cotidianas sin problema. En el Laboratorio de Empresa y la Empresa Experimental se atienden incontables sugerencias superficiales, que detienen momentáneamente la acción; sin embargo, la tendencia a continuar subsiste. La sugerencia puede o no ser aceptada y continuar; el conflicto se da cuando en ella se insiste, u otra idea le compite. Entonces, se producen nuevas ideas que serán mejoradas. Si hay tiempo para el pensamiento reflexivo, se hace evidente la importancia de la vacilación o postergación necesaria para la observación de los medios y su correlación con los objetivos.

El pensamiento vuelve sobre sí mismo para examinar la finalidad de la idea, con respecto a la condición de los medios disponibles y obstáculos a superar. El Laboratorio de Empresa incluido transversalmente en el plan de estudios, permite graduar la acción de la pedagogía activa, inmersa en una didáctica articulada con orientación educativa constructivista (Londoño, 1998).

\section{Segunda Etapa: La intelectualización}

Concebir, armar o intelectualizar el problema no es tarea fácil. En el ambiente empresarial hay una cantidad de hechos que generan tensión velada. El Laboratorio 
de Empresa presenta dos estados complementarios: a) Si de antemano en la Empresa Experimental todos observaran y razonaran el problema en la misma forma, ya se habría avanzado parte del camino que hay que clarificar y recorrer; b) Si todos los estudiantes, profesores y terceros sugirieran al mismo tiempo, con la misma rapidez y simultáneamente los mismos o similares razonamientos sería extraordinario, pero la realidad es otra. Para todos, inicialmente la comprensión de las situaciones problemáticas también es vaga y provisional, y adicionalmente, cada semestre, se vuelven a iniciar todas las reflexiones y experimentos, con distintos estudiantes y entorno relativamente modificado, no parametral, "...donde transitan los sentidos y los significados, es decir la realidad" (Quintar, 2008, pág. 42).

Al observar la Empresa Experimental, el pensamiento hace un inventario de medios, insinúa soluciones, despeja inconsistencias e intelectualmente, define el problema. Ante una sugerencia bloqueada, el pensamiento nuevamente analiza medios, ayudas, obstáculos o dificultades e inspecciona las condiciones de la Empresa Experimental. Cuando la dificultad es localizada se convierte en un problema intelectual, se define y deja de ser un fastidio incomprensible (Dewey, 1998). El semestre siguiente, vuelven y juegan las observaciones y razonamientos de los nuevos participantes.

Las instituciones que asumen la educación empresarial necesitan generar didáctica activa, que permita rescatar la acción reflexiva sobre la imaginada. En primera instancia, hay que detectar los conflictos de aprendizaje e intelectualización de los problemas relacionados con la teoría, o su uso y la comprensión en la Empresa Experimental. El estudiante y el docente pueden producir una actividad reflexiva, que partiendo de una realidad, logren una reconstrucción cognitiva en contexto. Intelectualizar experimentos en el Laboratorio de Empresa, requiere dosificar la acción pedagógica en la sencillez de la compleja totalidad de la Empresa Experimental. Responde como referente intelectual y práctico, progresivo del estudiante, concordante con el desarrollo académico del plan de estudios y transferencia verificable de conocimientos, llevado a una empresa real.

\section{Tercera Etapa: de la idea a la hipótesis}

Desde la primera sugerencia de la etapa I, aparecen en la mente las ideas para la acción, pero no hay control sobre ella. El repaso de medios e ideas clarifican el camino y amplían las opciones a seguir. La acción dosificada admite tomar forma a la idea y técnicamente da paso a la hipótesis.

La idea así planteada y controlada, con nuevas reflexiones, va pasando de lo provisional a lo definitivo. Surgen nuevos datos o hechos en movimiento que coinciden con la idea seleccionada. La decisión adoptada deja de ser una simple sugerencia y se convierte en una decisión verificable con los datos de la Empresa Experimental, que permite medir y programar nuevas experiencias en el laboratorio.

Unas sugerencias y luego decisiones empresariales, que son producto de observaciones completas, acompañadas de la totalidad del material perceptible y abstracto, articulan muchas causas y prescriben diversas consecuencias. De las sugerencias del Laboratorio de Empresa respecto a las hipótesis sugeridas por la observación y razonamiento de profesores y estudiantes, las decisiones son potestad exclusiva del cuerpo directivo de la Empresa Experimental, quienes sugerirán, aceptarán, aplazarán o rechazarán el uso de la hipótesis planteada inicialmente. 


\section{Cuarta Etapa: razonamiento generador de hipótesis}

En casos complejos se presentan muchas cadenas de conceptos que relacionan y producen nuevas ideas. Este encadenamiento sucesivo no sólo depende del arsenal educativo, cultural y científico del grupo o persona generadora de una hipótesis. El desarrollo científico, tecnológico, técnico, la época y el lugar en que se desenvuelve la Empresa Experimental, también intervienen como factores razonantes.

El desarrollo de ideas complejas mediante el razonamiento, proporciona términos intermedios con ideas que antes parecían elementos separados e irreconciliables. Los ambientes completos producen ideas fructíferas y otras ideas antagónicas. La mente empresarial trae ciertas razones y origina contradicciones o deducciones opuestas, totalmente respetables. La toma de decisiones a implementar, no sólo debe estar amparada en la imaginación, sino acompañada de medios para su aplicación.

El material forjado por la Empresa Experimental y los medios disponibles al estudiante, propician hechos y conceptos contextualizados al ambiente experimental de la IE afín al conocimiento empresarial. Facilitan la generación de eslabones y encadenamiento sucesivo de ideas razonadas como ejercicio académico, sin que sea forzoso aplicar. La capacidad de encadenar datos, ideas y nuevos eslabones sugeridos para el razonamiento, clarifican el pensamiento en complejos y potencian el concepto verdadero.

\section{Quinta Etapa: Comprobación de hipótesis}

La etapa final, es la demostración de muchos razonamientos. Para las pruebas como investigación formativa en el Laboratorio de Empresa, se han de organizar experiencias transversales al currículo, basados en datos controlables de la Empresa Experimental, para la actividad reflexiva experimental. La idea cuasi experimental, pre experimental o la exploración, sugieren nuevas hipótesis.

Se ha iniciado con el contraste de lo mínimo normativo, al superar las excusas para la verificación empírica de la teoría organizacional, con acción y participación didáctica afín a la pedagogía activa. Si se garantiza el acercamiento de la teoría a la práctica de la disciplina empresarial, se aprovechan las oportunidades posteriores en escenarios organizacionales más complejos. Por lo tanto, el pensamiento reflexivo para temas empresariales, debe contar con ambientes al máximo reconocibles por la observación de hechos controlables, que contrarresten el efecto de las variables inobservables. Solo así se encadenan sugerencias y eslabonan soluciones como producto de un razonamiento científico.

\section{CONCLUSIONES}

La educación empresarial debe acercar teoría y práctica, inicialmente con investigación formativa. Luego si tratar los subsecuentes conflictos de la formación: en la práctica de fin de carrera, en la profesión y en la empresa.

La teoría organizacional básica se probará en cada IE que acepte desafíos didácticos activos, o por lo menos, experimenten el conocimiento normativo. Aquí, se presentan dos opciones: aflorarán las tensiones, se nubla el pensamiento y se detiene la acción, o se analiza medios, ayudas adecuadas y producen nuevas ideas correlativas 
al objetivo. Las sugerencias, ahora dejan ver aristas controlables, con relación a otros hechos sin control o desconocidos. La hipótesis en los sistemas dinámicos en torno a una totalidad, se da como un razonamiento verdadero y verificable.

Se plantea consolidar un Laboratorio de Empresa que analice los medios y estructure una didáctica con pedagogía activa, capaz de dosificar la acción en cada IE. Se conoce que los hechos de la Empresa Experimental son reales y su presencia o ausencia, puede generar incomodidad para contrastar la teoría en la IE. No superar la falencia didáctica transversal a la formación en el tema empresarial, hace que el dilema siga.

La práctica activa transversal al plan de estudios pasa del uso de simples instrumentos, recursos fragmentados, e inadecuados, a una didáctica organizacional activa, confrontada en el Laboratorio de Empresa desde la IE. Fortalece el desarrollo de propuestas como la "Estrategia nacional de educación económica y financiera" (MHCP, 2010), inicia el proceso de formación del concepto y verifica unas teorías de Taylor, Fayol y muchas otras.

El Laboratorio de Empresa, es un desafío que insta a unir teoría y práctica de ese conocimiento, inicialmente en la Empresa Experimental, como también lo han hecho varias IE de educación media del área de influencia de la Uptc (Bernal, 2016).

\section{REFERENCIAS BIBLIOGRÁFICAS}

Agudelo, N. (2007). Sentido de la formación de Administradores(as) en la educación superior en Colombia. (Tesis de doctorado) Universidad del Cauca, Popayán.

Aktouf, O. (13 de noviembre de 2000). La Administración y su enseñanza ¿entre doctrina y ciencia? Administración y Pedagogía, 21-22.

Barnechea, M., González, E., \& Morgan, M. (junio de 1994). La Sistematización como Producción de Conocimiento. Taller permanente de Sistematización CEAAL., 1-10.

Bernal, H. (1997). Formalización del área de sistemas en la carrera de Administración de Empresas. (Tesis de Maertría) Fundación Universidad Central, Bogotá D.C.

Bernal, H. (2016). El Laboratorio de Empresa como Modelo Didáctico de Formación Multidisciplinar. (Suficiencia Investigativa de doctorado) Universidad Pedagógica y Tecnológica de Colombia, Tunja.

Bernal, H. (2018). Memorias Encuentro Internacional de Investigadores en Administración 2018 Noviembre 20 y 21 Cali, Colombia. (Universidad Externado de Colombia, Editor, \& Administración de Empresas) Obtenido de http://administracion.uexternado.edu.co/encuentroinvestigacion/ plantillas/2018/MemoriasEncuentroInvestigacion2018.pdf

Bernal, H., \& Berdugo, D. (1985). Programa de Práctica Integral. (Tesis de pregrado), Universidad Pedagógica y Tecnológica de Colombia, Tunja. 
Chiavenato, I. (2007). Introducción a la teoría general de la administración. México D.F.: McGrawHill.

CNA. (1996). Lineamientos para la acreditación de programas de pregrado. (2 ed.). Bogotá D.C.: Corcas Editores Ltda.

CNA. (2013). Lineamientos para la acreditación de programas de pregrado. Bogotá D.C.: CNA.

Colciencias. (2013). Recuperado el 18 de 8 de 2014, de http://201.234.78.173:8083/ciencia-war/ BusquedaGrupoXArea.do

Colciencias. (2015a). GrupLAC en Línea. Recuperado el 14 de mayo de 2017, de http://scienti. colciencias.gov.co:8080/gruplac/.

Colciencias. (2015b). Modelo de medición de grupos de investigación, desarrollo tecnológico o de innovación y de reconocimiento de investigadores del sistema nacional de ciencia, tecnología e innovación, año 2015. Bogotá: Colciencias.

Colombia. Ley 30. (1992). Por el cual se organiza el servicio público de la Educación Superior. 28 de diciembre de 1992.

CPAE. (2013). La Administración del Siglo XXI. Perspectivas para el fortalecimiento de la profesión en Colombia. Bogotá D.C.: UNAL.

CPAE. (2017). Tendencias Curriculares en la Educación Profesional de Administración de Empresas. Bucaramanga: Universidad Francisco de Paula Santander.

Dewey, J. (1998). Cómo pensamos. Nueva exposición de la relación entre pensamiento y proceso educativo. Barcelona: Paidos.

Díaz, Á. (1998). La Investigación en el campo de la Didáctica. Modelos Históricos. Perfiles educativos(80), 23.

Gallego, R., \& Pérez, R. (1997). La enseñanza de las ciencias experimentales, El constructivismo del caos. Bogotá D.C.: Cooperativa Editorial Magisterio.

García, A., \& Malaver, F. (2000). Una propuesta de pedagogía para un departamento deadministración. Cuadernos de Administración, (22), 83-104.

Ghiso, A. (Junio de 2004). http://centroderecursos.alboan.org/ebooks/0000/0416/Aportes57. pdf. (E. D. Educativa., Editor) Recuperado el 11 de 2015, de http://centroderecursos.alboan.org/ ebooks/0000/0416/Aportes57.pdf

Hernández, R., Fernández, C., \& Baptista, M. d. (2010). Metodología de la investigación. México: McGraw Hill.

ICFES. (diciembre de 1996). Población estudiantil por programas académicos 1995. (Base de Datos).

ICFES. (1 de agosto de 2002). A identificar la U. de alto riesgo. El Tiempo, págs. 2-9. 
Jara, Ó. (Febrero de 2012). http://educacionglobalresearch.net/wp-content/uploads/O2A-JaraCastellano.pdf. (E. Global, Editor, i. y. Sistematización de experiencias, Productor, \& REVISTA INTERNACIONAL sobre INVESTIGACIÓN en EDUCACIÓN GLOBAL y para el DESARROLLO) Recuperado el 25 de Agosto de 2014, de http://educacionglobalresearch.net/wp-content/ uploads/02A-Jara-Castellano.pdf

Kemmis, S., \& McTaggart, R. (1992). Cómo Planificar la Investigación Acción. Barcelona: Laertes. Koontz, H., O’Donnell, C., \& Weihrich, H. (1986). Administración (8 ed.). México, D.F., México: McGraw Hill.

Koontz, H., Weihrich, H., \& Mark, C. (2012). Administración Una perspectiva global y empresarial (14 ed.). México D.F.: McGraw Hill.

Laufer,M.(2006).Falsas dicotomiasy dualidadesen ciencia.ProQuest. Recuperadoel2 de juniode2013, de http://search.proquest.com/docview/748394121/13E6A6256AB28D03B1/19?accountid=43790

Lewin, K. (1946). Action Research and Minority Problems. (A. R. Problems, Productor) Recuperado el 29 de abril de 2017, de http://www.cscd.osaka-u.ac.jp: http://www.cscd.osaka-u.ac.jp/user/ rosaldo/K_Lewin_Action_research_minority_1946.pdf

Londoño, C. (1998). Orientaciones Pedagógicas del Constructivismo. Pensamiento \& Acción,(2 y 3), 133-142.

Londoño, C. (2008). Avatares del Constructivismo: de Kant a Piaget. Historia de la Educación Latinoamericana, (10), 73-96.

López, D., \& Sanabria, N. (2011). La Práctica Pedagógica Investigativa en la Facultad de Educación de la Uptc. Seminario permanente de Pedagogía, (8), 59.

López, F. (2005). Doxa y Episteme en la Generación del Conocimiento Administrativo. Investigación en Administración en América Latina: Evolución y Resultados, 44-47.

Malaver, F. (2016). La internacionalización de La publicación Colombiana en el campo de La administración: avances y desafíos. Cuadernos de Administración, (52), 53-81.

Malaver, F., \& López, F. (2016). La investigación sobre la administración en Colombia (1965-2015): balance y perspectivas. Cuadernos de Administración, (52), 141-166.

Max-Neef, M. (2013). Educacion y Valores del Espiritu. Recuperado el 19 de abril de 2017, de https:// www.max-neef.cl/descargas/Max_Neef-Educacion_Y_valores_del_espiritu.pdf

Medina, A., \& Salvador, F. (2009). Didáctica General (2 ed.). Madrid: Pearson Prentice Hall.

Mejía , M. (2013). La Sistematización Empodera y produce saber y conocimiento sobre la práctica desde la propuesta para sistematizar la experiencia de habilidades para la vida. Bogotá Colombia: Linotipia Bolívar, Ediciones Desde Abajo.

MEN. (21 de 7 de 1993). Decreto 1403. Por el cual se reglamenta la ley 30 de 1992. Colombia. Recuperado el 1 de 5 de 2017, de http://www.mineducacion.gov.co/1621/articles-86265_archivo_ pdf.pdf 
MEN. (2002a). Por el cual se establece el crédito académico como mecanismo de evaluación de calidad,... Decreto 0808. Bogotá D.C.

MEN. (2002b). Por el cual se establecen estándares de calidad en programas profesionales de pregrado en Administración. Decreto 0938. Bogotá D. C.

MEN. (2003a). Por el cual se establecen las condiciones mínimas de calidad y demás requisitos para el ofrecimiento y desarrollo de programas académicos de educación superior y se dictan otras disposiciones. Decreto 2566. Bogotá D.C.

MEN. (2003b). Por la cual se definen las características específicas de calidad para los programas de pregrado en Administración. Resolución Número 2767. Bogotá D.C.

MEN. (2006). No a las universidades de 'garaje'. (Centro Virtual de Noticias - CVN) Recuperado el 13 de julio de 2013, de http://www.mineducacion.gov.co/cvn/1665/article-115461.html

MEN. (2012). Perfil académico y condiciones de empleabilidad de los graduados de educación superior 2002 - 2011. Recuperado el 13 de 7 de 2013, de http://www.graduadoscolombia.edu.co/ html/1732/articles-195072_perfil_2012.pdf

MEN. (2015). Por medio del cual se expide el Decreto Único Reglamentario del Sector Educación. Decreto No. 1075. Bogotá D.C.

MEN. (2016). Programas: Carreras ADMINISTRACION. Recuperado el 11 de 5 de 2017, de http:// bi.mineducacion.gov.co:8380/eportal/web/men-observatorio-laboral/programas-carreras1

MHCP. (2010). Estrategia nacional de educación económica y financiera (EEF). Colombia.

Montoya, R. (7 de febrero de 2010). En búsqueda de profesionales. El Tiempo, pág. 4.

Morín, E. (2004). La epistemología de la complejidad. Recuperado el 22 de julio de 2013, de https:// dialnet.unirioja.es/servlet/articulo?codigo=1001254

Palomar, R. (6-8 de Noviembre de 1986). La Preferencia por los Estudios de Administración de Empresas en Colombia. (Guadalupe Ltda., Ed.) ICFES Serie Memorias de Eventos Científicos Colombianos,, 41(41), 13 - 50 .

Piaget, J. (1987). Psicología y Pedagogía. Barcelona: Ariel S.A.

Pinzón, C. (2016). Puentes entre la teoría y la práctica: la experimentación de las medidas descriptivas con el laboratorio de empresa. Recuperado el 14 de noviembre de 2016, de http://administracion.uexternado.edu.co/encuentroinvestigacion/plantillas/2016/ MemoriasEncuentroInvestigacion2016.pdf

Quintar, E. (2008). Didáctica no parametral: sendero hacia la descolonización. Ipecal, (224), 48.

Ramírez, M. (1994). La práctica: una aproximación al mundo empresarial. Memorias. V Encuentro Nacional de prácticas universitarias empresariales, 4-17. 
Ríos, J. (2008). Relevancia del estudio del lenguaje teórico sobre las organizaciones. Cuadernos de administración,, 21(37), 37 - 60.

Romero, J., Castelblanco, D., \& Bastidas, M. (2013). Diagnóstico del área financiera del sector supermercados e hipermercados en el departamento de Boyacá. Inquietud Empresarial, 13(2), 33-52.

Salinas, O. (1997). Objeto y método en la ciencia de la empresa. Memorias de ASCOLFA. (12). Papers ESADE.

SALVAT. (1968). Diccionario Enciclopédico, (Vol. 4, 3150). Barcelona: Salvat Editores S.A. Vygotsky, L. (1995). Pensamiento y Lenguaje. Buenos Aires: Ediciones Fausto.

Zambrano, A. (2006). Los hilos de la Palabra: Pedagogía y Didáctica. Bogotá D.C.: Cooperativa Editorial Magisterio.

Zemelman, H. (2009). Uso Crítico de la Teoría En torno a la función analítica de la totalidad. México D.F.: Instituto Politécnico Nacional. 